\title{
The effect of cigarette smoking on the clinical course of inflammatory bowel disease
}

\author{
Jacek Karczewski, Barbara Poniedziałek, Piotr Rzymski, Anna Rychlewska-Hańczewska, Zygmunt Adamski, \\ Krzysztof Wiktorowicz \\ Department of Biology and Environmental Sciences, Laboratory of Transplant Immunology, Poznan University \\ of Medical Sciences, Poznan, Poland
}

Prz Gastroenterol 2014; 9 (3): 153-159

DOI: $10.5114 / p g .2014 .43577$

Key words: inflammatory bowel disease, Crohn's disease, ulcerative colitis, smoking.

Address for correspondence: Jacek Karczewski MD, PhD, Department of Biology and Environmental Sciences, Laboratory of Transplant Immunology, Poznan University of Medical Sciences, 10 Fredry St, 61-701 Poznan, Poland, phone: +48 696822 816, fax: +48 618427551 , e-mail: jacek_karczewski@yahoo.com

\begin{abstract}
Introduction: Cigarette smoking is considered an important risk factor for developing Crohn's disease (CD), contributing to a more severe course of the disease. Conversely, smoking is believed to have a beneficial effect on the course of ulcerative colitis (UC), a second major condition of inflammatory bowel disease (IBD).

Aim: To investigate the effect of tobacco use on the clinical course of IBD.

Material and methods: A group of 95 adults with IBD were enrolled to the study. Demographic and clinical data of patients as well as their smoking status were analysed based on their medical history. Values were considered significant when $p \leq 0.05$.

Results: Current smokers constituted the majority of CD patients. They tended to develop a more severe course of the disease, compared to former smokers and non-smokers. Current smokers suffered a moderate-to-severe form of the disease and required immunosuppressive therapy more frequently. They were also hospitalised and underwent surgeries more frequently than patients from other investigated subgroups. The study failed, however, to fully confirm the beneficial effect of smoking on the clinical outcome of UC. The investigated non-smokers and former smokers suffered a more severe disease, but the analysis did not find any statistical differences in the frequencies of hospitalisations nor immunosuppressant usage among the investigated subgroups.

Conclusions: The study confirmed a detrimental effect of smoking on the outcome of CD, but failed to fully confirm its beneficial effect on UC.
\end{abstract}

\section{Introduction}

Inflammatory bowel disease (IBD), comprising primarily Crohn's disease (CD) and ulcerative colitis (UC), are characterised by chronic relapsing inflammation of the gastrointestinal tract. The precise aetiology of IBD remains largely unknown. Undoubtedly, IBD is of multifactorial nature, involving genetic predispositions, environmental factors, intestinal microbial flora and the immune system [1]. In spite of sharing many clinical features like abdominal pain, diarrhoea, weight loss and increased risk of developing colorectal cancer, the two diseases differ in location, extension and histological findings [2]. One of the most striking distinctions, however, is the link to smoking habits [3]. Several studies have demonstrated a strong association of smoking with $C D$, showing its detrimental effect on the course of the disease [4]. The meta-analysis performed by Calkins yielded a pooled odds ratio (OR) 2.0 (1.65-2.47) in current smokers and OR 1.80 (1.33-2.51) in former smokers, compared to life-time non-smokers [5]. Various reports are consistent about this effect, showing that about $50 \%$ of CD patients are smokers, mostly young women [4]. It also seems that smoking might determine disease location, with a higher prevalence of ileal disease and a lower prevalence of colonic involvement in smokers [6]. In contrast, UC has been reported to be associated with non-smokers and former smokers, and smoking seems to have a beneficial effect on the course of the disease [4]. The risk of developing UC is lower in current smokers compared to lifetime non-smokers 
with OR $0.41(0.34-0.48)$ [5]. The course of disease seems to be also milder in smokers than non-smokers. Paradoxically, former smokers are approximately 1.7 times more likely to develop UC than those who never smoked, and they also have a poorer disease course [5]. The reason why smoking has opposite effects on two diseases that share so many similarities remains unknown. A better understanding of the mechanism responsible for these differences would help to gain knowledge about the aetiology of IBD.

\section{Aim}

This study aimed to investigate the effect of smoking on the clinical outcome of IBD.

\section{Material and methods}

The project was approved by the Ethics Committee at Poznan University of Medical Sciences. A group of 95 adults with CD and UC, comprising 59 females (62.1\%) and 36 males (37.9\%) with a mean age of $35.5 \pm 7.2$ years (median: 35.8$)$, was enrolled. A group of CD patients included 55 adults, 32 females (58.2\%) and 23 males $(41.8 \%$ ) with a mean age of $31.8 \pm 5.8$ (median: 33.0) years, whereas a group of UC patients included 27 females $(67.5 \%)$ and 13 males (32.5\%) with a mean age $40.5 \pm 5.8$ (median: 39.7 ) years. All patients were Caucasian. The disease had been diagnosed and confirmed by endoscopic and radiologic means. For CD, disease behaviour and location were determined according to the Vienna classification [7]. For UC, disease activity was estimated using ECCO classification, and disease location was defined as proctitis, left-sided colitis and pancolitis [8]. Extraintestinal manifestations (EIM), including primary sclerosing cholangitis, were also identified. Only patients treated with conventional therapies, including 5-aminosalicylates, prednisolone, budesonide, azathioprine, mercaptopurine, methotrexate and antibiotics, were recruited to the study. The smoking status of patients was analysed based on medical history, and patients were divided into three subcategories: "current smokers", "former smokers" and "non-smokers". Current smokers were defined as patients smoking more than 1 cigarette per day within 6 months prior to the diagnosis. Former smokers were defined as patients who quit smoking more than 6 months prior to the diagnosis. Non-smokers were considered to have never smoked.

\section{Statistical analysis}

Descriptive variables are presented as means and medians with range, and categorical variables as frequencies with percentage. Frequencies were compared with $\chi^{2}$ test and means between two groups with the
Mann-Whitney test, and between three or more groups with the Kruskal-Wallis test using SPSS version 15. Values were considered significant when $p \leq 0.05$.

\section{Results}

\section{Characteristics of patients with inflammatory bowel diseases}

The characteristics of 95 patients with IBD participating in the study are presented in Table I. The group included 59 females (62.1\%) and 36 males (37.9\%) with a mean age of $35.5 \pm 7.2$ (median: 35.8 ) years. Seventy-one patients $(74.7 \%)$ in this group had been diagnosed before the age of 40 years (early onset): 51 patients $(53.7 \%)$ with CD and 20 patients $(21.0 \%)$ with UC $(p<0.001)$. The gender distribution between the CD and UC groups was similar, with a predominance of female patients in both groups (58.2\% vs. $67.5 \%$; $p>0.05)$. Medical history reviled that 17 CD patients (30.9\%) and 13 UC patients (32.5\%) had a family history of IBD (31.6\%). The majority of CD patients were current smokers $(60.0 \%)$, particularly young females with early onset (36.4\%), while the UC group was dominated by former smokers (40.0\%) and non-smokers (35.0\%), most of whom were also females (50.0\%). The most frequent locations of CD were: colon (36.4\%), terminal ileum (30.9\%), ileocolon (27.3\%) and upper gastrointestinal (GI) tract (5.4\%). As for CD behaviour, 26 patients $(47.3 \%)$ were classified as B1 at diagnosis, 19 patients (34.5\%) as B3 and 10 patients (18.2\%) as B2. There were 16 patients $(40.0 \%)$ with pancolitis, 13 patients (32.5\%) with left-sided colitis and 11 patients $(27.5 \%)$ with proctitis in the UC group. According to ECCO disease activity, 4 patients (10\%) had mild UC, 21 patients (52.5\%) had moderate UC and 15 patients (37.5\%) had severe UC. The CD patients more frequently experienced extraintestinal manifestations (EIM) than UC patients (30.9\% vs. $10 \% ; p=0.01)$, especially current smokers (21.8\%). Hospitalisations were also more frequent among CD patients than UC patients (34.6\% vs. $20.0 \% ; p>0.05)$ although the difference between both groups was not statistically significant.

\section{Patients with Crohn's disease}

The relation between smoking behaviour and CD outcome is presented in Table II. The group of CD patients included 55 adults, 32 females (58.2\%) and 23 males (41.8\%) with a mean age of $31.8 \pm 5.8$ (median: 33.0 ) years at diagnosis. The mean duration of the disease since the diagnosis was $2.4 \pm 1.5$ (median: $2.0)$ years. An analysis of medical histories revealed that 33 patients $(60.0 \%)$ in this group, 21 female $(38.2 \%)$ and 12 male (21.8\%), were smokers; 9 patients (16.4\%), 
Table I. Characteristics of patients with inflammatory bowel diseases

\begin{tabular}{|c|c|c|c|}
\hline Characteristic & $\mathrm{CD}(n=55)$ & UC $(n=40)$ & Value of $p$ \\
\hline \multicolumn{4}{|l|}{ Gender, $n(\%)$ : } \\
\hline Female & $32(58.2)$ & $27(67.5)$ & NS \\
\hline \multicolumn{4}{|l|}{ Age at diagnosis [years]: } \\
\hline Mean \pm SD & $31.8 \pm 5.8$ & $40.5 \pm 5.8$ & $<0.001$ \\
\hline Median (range) & $33.0(19.0-45.0)$ & $39.7(31.2-56.3)$ & $<0.001$ \\
\hline Early onset (< 40 years), $n$ (\%) & $51(92.7)$ & $20(50.0)$ & $<0.001$ \\
\hline \multicolumn{4}{|l|}{ Duration of disease [years]: } \\
\hline Mean \pm SD & $2.4 \pm 1.5$ & $2.7 \pm 1.6$ & NS \\
\hline Median (range) & $2.0(0.0-5.5)$ & $2.3(0.0-6.0)$ & NS \\
\hline Family history of IBD, $n(\%)$ & $17(30.9)$ & $13(32.5)$ & NS \\
\hline \multicolumn{4}{|l|}{ Hospitalisation, $n(\%)$ : } \\
\hline 1 time & $22(40.0)$ & $17(42.5)$ & NS \\
\hline At least 2 times & $19(34.6)$ & $8(20.0)$ & NS \\
\hline \multicolumn{4}{|c|}{ Smoking behaviour at diagnosis, $n(\%)$ : } \\
\hline Smoker & $33(60.0)$ & $10(25.0)$ & 0.002 \\
\hline Former smoker & $9(16.4)$ & $16(40.0)$ & 0.001 \\
\hline Non-smoker & $13(23.6)$ & $14(35.0)$ & NS \\
\hline \multicolumn{4}{|l|}{ CD location, $n(\%)$ : } \\
\hline Terminal ileum & $17(30.9)$ & & \\
\hline Colon & $20(36.4)$ & & \\
\hline Ileocolon & $15(27.3)$ & & \\
\hline Upper GI tract & $3(5.4)$ & & \\
\hline \multicolumn{4}{|l|}{ CD behaviour, $n(\%)$ : } \\
\hline B1 & $26(47.3)$ & & \\
\hline B2 & $10(18.2)$ & & \\
\hline B3 & $19(34.5)$ & & \\
\hline EIM, $n(\%)$ & $17(30.9)$ & $4(10.0)$ & 0.01 \\
\hline \multicolumn{4}{|l|}{ UC location, $n(\%)$ : } \\
\hline Proctitis & & $11(27.5)$ & \\
\hline Left-sided & & $13(32.5)$ & \\
\hline Pancolitis & & $16(40.0)$ & \\
\hline \multicolumn{4}{|l|}{ ECCO classification, $n(\%)$ : } \\
\hline Mild & & $4(10.0)$ & \\
\hline Moderate & & $21(52.5)$ & \\
\hline Severe & & $15(37.5)$ & \\
\hline
\end{tabular}

B1 - non-constricturing, non-penetrating, B2 - structuring, B3 - penetrating, CD -Crohn's disease, ECCO - European Crohn's and Colitis Organisation, EIM - extraintestinal manifestations, IBD - inflammatory bowel diseases, NS - not significant, UC - ulcerative colitis, SD - standard deviation

4 female (7.3\%) and 5 male (9.1\%), were former-smokers; and 13 patients (23.6\%), 7 female (12.7\%) and 6 male (10.9\%), were non-smokers. Medical history did not show any change in smoking behaviour of patients during follow-up. Seventeen patients (30.9\%) in this group had a family history of $C D$, mostly non-smokers and former smokers, although the differences were not statistically significant. Current smokers, particularly young females (mean age: $30.8 \pm 6.4$ years), were the youngest at diagnosis, followed by former smokers and non-smokers $(p>0.05)$. In all investigated subgroups of $C D$ patients the onset of disease was observed earlier 
Table II. Characteristics of patients with Crohn's disease according to smoking behaviour $(n=55)$

\begin{tabular}{|c|c|c|c|}
\hline Characteristic & Smokers $(n=33)$ & Former smokers $(n=9)$ & Non-smokers $(n=13)$ \\
\hline \multicolumn{4}{|l|}{ Gender, $n(\%)$ : } \\
\hline Female & $21(63.6)$ & $4(44.4)$ & $7(53.8)$ \\
\hline \multicolumn{4}{|l|}{ Age at diagnosis [years]: } \\
\hline Mean \pm SD & $30.9 \pm 6.2$ & $31.5 \pm 4.7$ & $34.4 \pm 4.7$ \\
\hline Median (range) & $30.2(19.0-43.2)$ & $30.1(26.2-38.0)$ & $33.3(28.8-45.0)$ \\
\hline Early onset (< 40 years), $n$ (\%) & $31(93.9)$ & $9(100.0)$ & $11(84.6)$ \\
\hline \multicolumn{4}{|l|}{ Duration of disease [years]: } \\
\hline Mean \pm SD & $2.5 \pm 1.5$ & $2.6 \pm 1.7$ & $2.0 \pm 1.1$ \\
\hline Median (range) & $2.0(0.0-5.5)$ & $3.0(0.0-5.0)$ & $1.5(1.5-0.6)$ \\
\hline Family history of IBD, $n(\%)$ & $7(21.2)$ & $4(44.4)$ & $6(46.2)$ \\
\hline \multicolumn{4}{|l|}{ Hospitalization, $n(\%)$ : } \\
\hline 1 time & $12(36.4)$ & $3(33.3)$ & $7(53.8)$ \\
\hline At least 2 times & $13(39.4)$ & $3(33.3)$ & $3(23.1)$ \\
\hline \multicolumn{4}{|l|}{ Surgery, $n(\%)$ : } \\
\hline 1 time & $10(30.3)$ & $2(22.2)$ & $4(30.8)$ \\
\hline At least 2 times & $9(27.3)$ & $2(22.2)$ & $2(15.4)$ \\
\hline \multicolumn{4}{|l|}{ CD location, $n$ (\%): } \\
\hline Terminal ileum & $12(36.4)$ & $2(22.2)$ & $3(23.1)$ \\
\hline Colon & $10(30.3)$ & $5(55.6)$ & $5(38.5)$ \\
\hline Ileocolon & $11(33.3)$ & $2(22.2)$ & $2(15.3)$ \\
\hline Upper GI tract & $0(0.0)$ & $0(0.0)$ & $3(23.1), p=0.04$ \\
\hline 5-ASA, $n(\%)$ & $32(97.0)$ & $8(88.9)$ & $12(92.3)$ \\
\hline Glucocorticoids, $n(\%)$ & $26(78.8)$ & $6(66.7)$ & $10(76.9)$ \\
\hline Immunosuppressants, $n(\%)$ & $13(39.4)$ & $2(22.2)$ & $2(23.1)$ \\
\hline \multicolumn{4}{|l|}{ CD behaviour, $n(\%)$ : } \\
\hline B1 & $15(45.5)$ & $4(44.4)$ & $7(53.8)$ \\
\hline B2 & $3(9.0)$ & $2(22.2)$ & $5(38.5)$ \\
\hline B3 & $15(45.5)$ & $3(33.3)$ & $1(7.7), p=0.05$ \\
\hline EIM, $n(\%)$ & $13(39.4)$ & $1(11.1)$ & $3(23.1)$ \\
\hline \multicolumn{4}{|l|}{ CDAI, $n(\%):$} \\
\hline$<150$ & $3(9.1)$ & $4(44.4), p=0.05$ & $4(30.8), p=0.05$ \\
\hline $150-220$ & $17(51.5)$ & $2(22.3)$ & $5(38.4)$ \\
\hline $220-450$ & $12(36.4)$ & $3(33.3)$ & $4(30.8)$ \\
\hline$>450$ & $1(3.0)$ & $0(0.0)$ & $0(0.0)$ \\
\hline
\end{tabular}

5-ASA - 5-aminosalicylates, B1 - non-constricturing, non-penetrating, B2 - structuring, B3-penetrating, CD -Crohn's disease, CDAI-Crohn's disease Activity Index, EIM - extraintestinal manifestations, GI tract - gastrointestinal tract, $S D$ - standard deviation, ${ }^{*} p \leq 0.05 \mathrm{vs}$. smokers

in females than males although the differences were not statistically significant. Analysis showed that current smokers tended to have more severe course of the disease compared to former smokers and non-smokers. The highest frequency of hospitalisations during follow-up was observed in this subgroup (at least 2 hospitalisations: $13 ; 39.4 \%)$, especially among females $(8 ; 24.2 \%)$, as well as the highest frequency of surgeries (at least 2 surgeries: 9; 27.3\%). The highest rate of cases of moderate-to-severe disease, measured as CDAI $\geq 220$, was also found among current smokers (13; 24.2\%). Current smokers, particularly females, were also prescribed immunosuppressants more frequently than patients from other subgroups. On the contrary, the highest number of asymptotic patients with CDAI $<150$ was observed among former smokers (44.0\%) 
Table III. Characteristics of patients with ulcerative colitis according to smoking behaviour $(n=40)$

\begin{tabular}{|c|c|c|c|}
\hline Characteristic & Smokers $(n=10)$ & Former smokers $(n=16)$ & Non-smokers $(n=14)$ \\
\hline \multicolumn{4}{|l|}{ Gender, $n(\%)$ : } \\
\hline Female & $7(70.0)$ & $9(56.3)$ & $11(78.6)$ \\
\hline \multicolumn{4}{|l|}{ Age at diagnosis [years]: } \\
\hline Mean \pm SD & $41.0 \pm 7.6$ & $40.1 \pm 5.8, p=0.05$ & $40.5 \pm 4.7, p=0.05$ \\
\hline Median (range) & $41.2(31.2-56.3)$ & $38.3(32.1-55.0)$ & $39.6(32.1-47.3)$ \\
\hline Early onset ( $<40$ years), $n(\%)$ & $4(0.0)$ & $7(43.8)$ & $7(50.0)$ \\
\hline \multicolumn{4}{|l|}{ Duration of disease [years]: } \\
\hline Mean \pm SD & $1.8 \pm 1.1$ & $3.2 \pm 1.8, p=0.04$ & $2.8 \pm 1.4, p=0.05$ \\
\hline Median & 2.0 & 3.1 & 2.4 \\
\hline Family history of IBD, $n(\%)$ & $2(20.0)$ & $6(37.5)$ & $5(35.7)$ \\
\hline \multicolumn{4}{|l|}{ Hospitalization, $n(\%)$ : } \\
\hline 1 time & $3(30.0)$ & $7(43.8)$ & $7(50.0)$ \\
\hline At least 2 times & $2(20.0)$ & $3(18.8)$ & $3(21.4)$ \\
\hline Colectomy, $n(\%)$ & $1(10.0)$ & $3(18.8)$ & $2(14.3)$ \\
\hline 5-ASA, $n(\%)$ & $10(100)$ & $15(93.7)$ & $14(100)$ \\
\hline Glucocorticoids, $n(\%)$ & $6(60.0)$ & $8(62.5)$ & $4(28.6)$ \\
\hline Immunosuppressants, $n$ (\%) & $2(20.0)$ & $4(25.0)$ & $4(28.6)$ \\
\hline \multicolumn{4}{|l|}{ UC location, $n(\%)$ : } \\
\hline Proctitis & $2(20.0)$ & $5(31.3)$ & $4(28.6)$ \\
\hline Left-sided & $3(30.3)$ & $5(31.3)$ & $5(35.7)$ \\
\hline Pancolitis & $5(50.0)$ & $6(37.4)$ & $5(35.7)$ \\
\hline \multicolumn{4}{|l|}{ ECCO classification, $n$ (\%): } \\
\hline Mild & $3(30.0)$ & $0(0.0), p=0.01$ & $1(7.1), p=0.03$ \\
\hline Moderate & $6(60.0)$ & $9(56.2)$ & $6(42.9)$ \\
\hline Severe & $1(10.0)$ & 7 (43.8), $p=0.01$ & $7(50.0), p=0.03$ \\
\hline EIM, $n(\%)$ & $0(0.0)$ & $3(18.8)$ & $1(7.1)$ \\
\hline
\end{tabular}

and non-smokers $(30.8 \%)$, compared to the current smokers subgroup (9.1\%; $p<0.05)$. No statistical differences in CD location and behaviour were found among the investigated subgroups, except for the presence of disease in the upper Gl tract solely in non-smokers $(23.1 \% ; p=0.05)$ and rare disease behaviour B3 in this subgroup (7.7\%; $p=0.05)$. Fewer EIM were experienced by former smokers and non-smokers than by current smokers.

\section{Patients with ulcerative colitis}

The characteristics of UC patients according to smoking behaviour are presented in Table III. The group of 40 UC patients with a mean age of $40.5 \pm 5.8$ (median: 39.7$)$ years at diagnosis comprised 13 males (32.5\%) and 27 females (67.5\%), the majority of whom were former smokers and non-smokers. The mean duration of the disease since diagnosis was $2.7 \pm 1.6$ (median: 2.5 ) years. The prevalence of smoking at the time of IBD diagnosis was much lower in UC patients than in CD patients. Based on medical history, 10 patients (25.0\%), 7 females (17.5\%) and 3 males (7.5\%), were identified as current smokers; 16 patients (40.0\%), 9 females (22.5\%) and 7 males (17.5\%) were former smokers; and 14 patients (35.0\%), 11 females (27.5\%) and 3 males (7.5\%), were non-smokers. No change in patients' smoking behaviour was observed since the diagnosis. Thirteen patients (32.5\%) in this group, particularly former smokers (37.5\%) and non-smokers (35.7\%), had a family history of UC $(p>0.05)$. The majority of patients with a family history of UC in those subgroups were females $(9 ; 22.5 \%)$. Female patients also had an 
earlier onset of the disease compared to males, except for the current smoker subgroup $(p=0.05)$. Analysis showed that non-smokers and former smokers developed more severe course of the disease compared to current smokers. The highest rate of cases of severe disease, estimated according to ECCO classification, was found among non-smokers (50.0\%; $p=0.01$ ) and former smokers $(42.9 \%)(p=0.03)$. Analysis did not show any statistically significant differences in frequencies of immunosuppressant usage, hospitalisations, colectomies, EIM or location of UC, among the investigated subgroups.

\section{Discussion}

The presented data confirm the detrimental effect of smoking on the outcome of CD, but failed to fully confirm its beneficial effect on UC. Current smokers constituted the majority of CD patients (60\%), which is consistent with other reports [5]. They tended to develop a more severe course of the disease, compared to former smokers and non-smokers. They suffered a moderate-to-severe form of the disease (CDAI $\geq 220-450$ ) requiring immunosuppressive therapy more frequently $[9,10]$. The increased need for immunosuppressants among smokers is supposedly dose-dependent [11], although this aspect was not analysed in the study. Smokers were also hospitalised and underwent surgeries more frequently than patients from other investigated subgroups $[12,13]$. The deleterious effect of smoking in $C D$ is particularly manifested in patients undergoing surgeries. It has been reported that the cumulative rates of clinical and surgical recurrence are significantly more elevated in smokers than in non-smokers $[14,15]$. Cottone et al. have shown that macroscopic lesions on the ileal site of the anastomosis were observed 1 year after surgery in $70 \%$ of smokers vs. $35 \%$ of non-smokers and $27 \%$ of former smokers [14]. Due to the relatively short period of observation (mean: $2.4 \pm 1.5$ years; median: 2.0 years), this effect was not observed in the presented study. The study showed that smoking was preferentially associated with ileal disease, and non-smoking with colonic disease, although the observed differences were not statistically significant $[9,12]$. It is worth noting that all instances of disease location in the upper GI tract were found among non-smokers ( $p=0.04)$. Current smokers also experienced EIM more often than non-smokers and former smokers. Interestingly, in all investigated subgroups, particularly among current smokers, the female patients seemed to be more affected by $\mathrm{CD}$; they developed a more severe form of the disease and experienced the onset earlier than men. This observation is consistent with other reports [16]. According to some authors, gender differences in CD development vary geographically [17], and are strongly influenced by attained age, and are present after the mid-second decade of life [18]. A few possible explanations of this phenomenon have been proposed, like the negative effect of oestrogen exerted on proinflammatory cytokine gene regulation and immune cell interactions [19] or differences in smoking habits among genders, since females use more filter cigarettes and more light cigarettes, resulting in higher relative exposure to smoke than nicotine [20].

In contrast to most reports so far, the presented study failed to fully confirm the beneficial effect of smoking on the clinical outcome of UC. The prevalence of smoking at the time of IBD diagnosis was much lower in UC patients than in CD patients, which is consistent with other reports [5]. Investigated non-smokers and former smokers suffered more severe disease, estimated according to ECCO classification, than current smokers $(p<0.05)$. The analysis, however, did not show any statistical differences in the frequencies of hospitalisations nor immunosuppressant usage among the investigated subgroups, which has also been reported by some authors [19, 21-23]. It has also been implied that smoking significantly lowers the risk of colectomy. In a retrospective analysis of a large series of UC patients conducted by Mokbel et al., smoking was found to decrease the 10-year cumulative colectomy risk from 0.42 to 0.32 [23]. Also a meta-analysis of several large series of UC gave an odds ratio of 0.57 (95\% confidence intervals 0.38-0.85) for total colectomy in current smokers compared to non-smokers [24]. However, the presented data did not confirm these findings. Likewise, some previous studies have suggested the protective effect of smoking against the development of colonic disease [12, 25], whereas others have not found this association $[3,11]$. The presented study failed to find any association between smoking status of UC patients and disease location. Interestingly, the study demonstrated a greater prevalence of females in UC, which is in conflict with some previous reports [26].

The presented study had some limitations. The retrospective nature of the study may have led to some bias in the interpretation of data. A relatively small number of patients in the study could have contributed to unexpected and/or non-significant results. For example, the fact that there was no clear positive effect of smoking on the course of UC observed in the study could result from a lack of power considering the relatively small number of patients. It could be also related, however, to the relatively short period of observation (2.7 \pm 1.6 years; median 2.3 years), although it was not 
the case in CD. Also, the daily dose of nicotine and the effect of passive smoking were not analysed for the purposes of this study.

\section{Conclusions}

The study confirmed the detrimental effect of smoking on the outcome of $C D$, but failed to fully confirm its beneficial effect on UC. An interesting observation is the prevalence of female patients in both CD and UC, as well as their more severe course of $C D$.

\section{References}

1. Xavier RJ, Podolsky DK. Unravelling the pathogenesis of inflammatory bowel disease. Nature 2007; 448: 427-34.

2. Targan SR, Shanahan F, Karp LC. Inflammatory bowel disease: translating basic science into clinical practice. 5 ed. Wiley-Blackwell 2010; 752.

3. Benoni C, Nilsson A. Smoking habits in patients with inflammatory bowel disease. Scand I Gastroenterol 1984; 19: 824-30.

4. Birrenbach T, Böcker U. Inflammatory bowel disease and smoking: a review of epidemiology, pathophysiology, and therapeutic implications. Inflamm Bowel Dis 2004; 10: 848-59.

5. Calkins BM. A meta-analysis of the role of smoking in inflammatory bowel disease. Dig Dis Sci 1989; 34: 1841-54.

6. Cosnes J. What is the link between the use of tobacco and IBD? Inflamm Bowel Dis 2008; Suppl 2: S14-5.

7. Gasche C, Scholmerich J, Brynskov J, et al. A simple classification of Crohn's disease: report of the Working Party for the World Congresses of Gastroenterology, Vienna 1998. Inflamm Bowel Dis 2000; 6: 8-15.

8. Stange EF, Travis SP, Vermeire S, et al. European evidence-based Consensus on the diagnosis and management of ulcerative colitis: definitions and diagnosis. J Crohn's Colitis 2008; 2: 1-23.

9. Lindberg E, Järnerot G, Huitfeldt B. Smoking in Crohn's disease: effect on localisation and clinical course. Gut 1992; 33: 779-82.

10. Cosnes J, Carbonnel F, Beaugerie L, et al. Effects of cigarette smoking on the long-term course of Crohn's disease. Gastroenterology 1996; 110: 424-31.

11. Tobin MV, Logan RF, Langman MJ, et al. Cigarette smoking and inflammatory bowel disease. Gastroenterology 1987; 93: 316-21.

12. Russel MG, Volovics A, Schoon EJ, et al. Inflammatory bowel disease: is there any relation between smoking status and disease presentation? European Collaborative IBD Study Group. Inflamm Bowel Dis 1998; 4: 182-6.

13. Breuer-Katschinski BD, Holländer N, Goebell H. Effect of cigarette smoking on the course of Crohn's disease. Eur J Gastroenterol Hepatol 1996; 8: 225-8.

14. Cottone M, Rosselli M, Orlando A, et al. Smoking habits and recurrence in Crohn's disease. Gastroenterology 1994; 106: 643-8.

15. Yamamoto T, Keighley MR. The association of cigarette smoking with a high risk of recurrence after ileocolonic resection for ileocecal Crohn's disease. Surgery Today 1999; 29: 579-80.
16. Sutherland LR, Ramcharan S, Bryant H, Fick G. Effect of cigarette smoking on recurrence of Crohn's disease. Gastroenterology 1990; 98: 1123-8.

17. Brant SR, Nguyen GC. Is there a gender difference in the prevalence of Crohn's disease or ulcerative colitis? Inflamm Bowel Dis 2008; 14: S2-3.

18. Molinié F, Gower-Rousseau C, Yzet T, et al. Opposite evolution in incidence of Crohn's disease and ulcerative colitis in Northern France (1988-1999). Gut 2004; 53: 843-8.

19. Rider V, Abdou NI. Gender differences in autoimmunity: molecular basis for estrogen effects in systemic lupus erythematosus. Int Immunopharmacol 2001; 1: 1009-24.

20. Zeman MV, Hiraki L, Sellers EM. Gender differences in tobacco smoking: higher relative exposure to smoke than nicotine in women. J Women's Health Gender Based Med 2002; 11: 147-53.

21. Moum B, Ekbom A, Vatn MH, et al. Clinical course during the 1st year after diagnosis in ulcerative colitis and Crohn's disease. Results of a large, prospective population-based study in southeastern Norway, 1990-93. Scand J Gastroenterol 1997; 32: 1005-12.

22. Boyko EJ, Perera DR, Koepsell TD, et al. Effects of cigarette smoking on the clinical course of ulcerative colitis. Scand J Gastroenterol 1988; 23: 1147-52.

23. Mokbel M, Carbonnel F, Beaugerie L, et al. Effect of smoking on the long-term course of ulcerative colitis. Gastroentorologie Clinique et Biologique 1998; 22: 858-62.

24. Cosnes J. Tobacco and IBD: relevance in the understanding of disease mechanisms and clinical practice. Best Pract Res Clin Gastroenterol 2004; 18: 481-96.

25. Holdstock G, Savage D, Harman M, Wright R. Should patients with inflammatory bowel disease smoke? Br Med J 1984; 288 : 362.

26. Bernstein CN, Blanchard JF, Rawsthorne P, Wajda A. Epidemiology of Crohn's disease and ulcerative colitis in a central Canadian province: a population-based study. Am J Epidemiol 1999; 15: 916-24.

Received: $\quad 10.10 .2013$

Accepted: 14.01.2014 